\title{
CRYSTAL STRUCTURE OF ZINC(II) 4,4'-OXYBIS(BENZOATE)
}

\author{
Jun Tao, ${ }^{*}$ Xin Yin, Rong-Bin Huang and Lan-Sun Zheng* \\ School of Chemistry and Chemical Engineering, Xiamen University, 361005 Xiamen, China
}

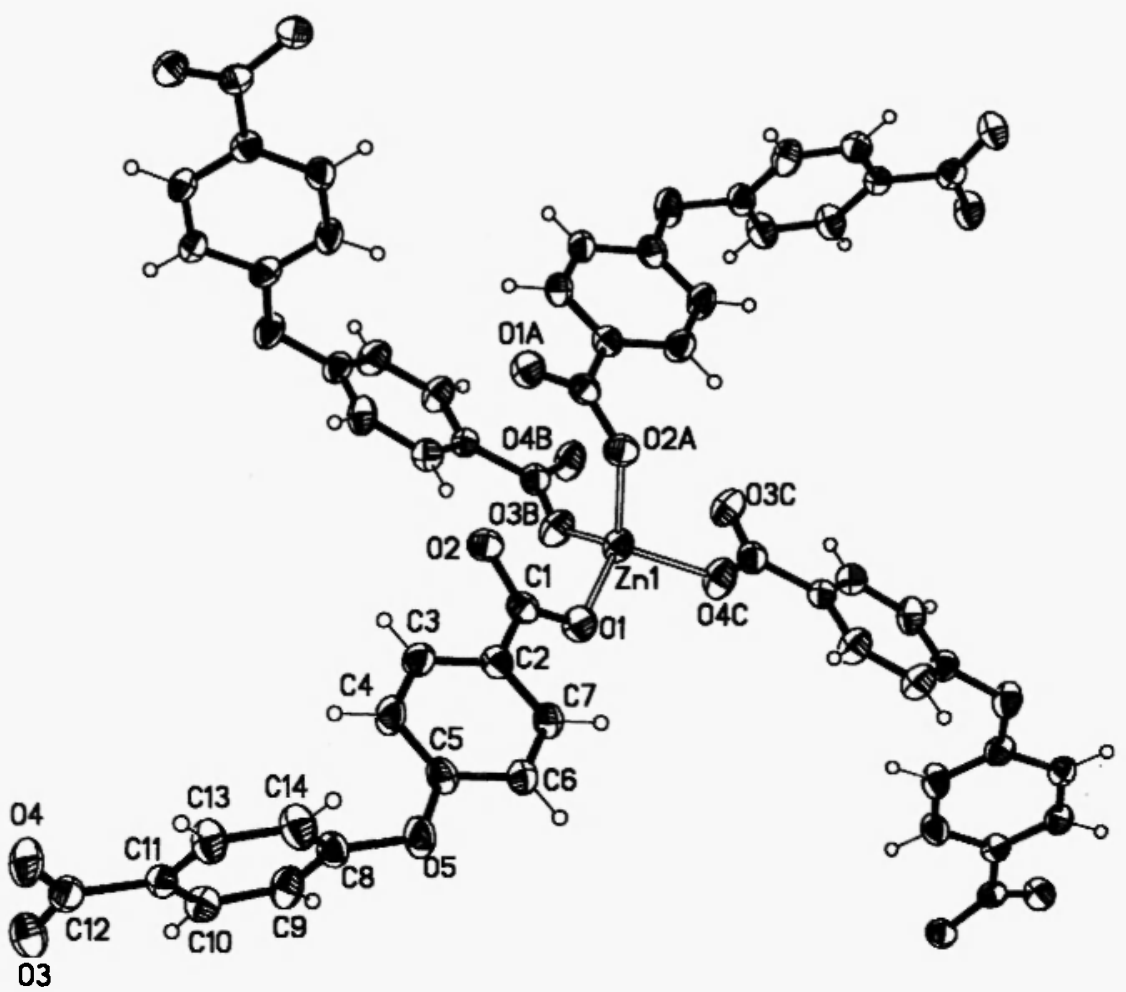

Figure 1. ORTEP plot of the title complex at the $50 \%$ probability level. Selected bond distances and angles: $\mathrm{ZnI}-\mathrm{Ol}=1.971(3), \mathrm{ZnI}-\mathrm{O} 2 \mathrm{~A}=1.978(3), \mathrm{ZnI}-\mathrm{O} 3 \mathrm{~B}=1.953(3), \mathrm{ZnI}-\mathrm{O} 4 \mathrm{C}=1.945(3) \AA$ $\mathrm{Ol}-\mathrm{ZnI}-\mathrm{O} 2 \mathrm{~A}=105.7(1), \mathrm{Ol}-\mathrm{ZnI}-\mathrm{O} 3 \mathrm{~B}=114.1(1), \mathrm{Ol}-\mathrm{ZnI}-\mathrm{O} 4 \mathrm{C}=103.3(1), \mathrm{O} 2 \mathrm{~A}-\mathrm{ZnI}-\mathrm{O} 3 \mathrm{~B}$ $=107.8(1), \mathrm{O} 2 \mathrm{~A}-\mathrm{Znl}-\mathrm{O} 4 \mathrm{C}=110.0(1), \mathrm{O} 3 \mathrm{~B}-\mathrm{Znl}-\mathrm{O} 4 \mathrm{C}=115.4(2)^{\circ}$. Symmetry transformation: $a=1$ $-x,-y, 1-z ; b=3 / 2-x, y-1 / 2,1 / 2-z, c=x-3 / 2,1 / 2-y, 1 / 2+z$.

\section{COMMENT}

Metal-organic frameworks based on rigid linear dicarboxylate linkers have attracted considerable attention because of their intrinsic aesthetic appeal and potentially exploitable properties [1]. More recently, rigid angular dicarboxylate linkers with a $120^{\circ}$ angle [2] and a $90^{\circ}$ angle [3] between two functional groups have been used to construct discrete nanometer-sized polygons and nanoscopic rectangles, respectively. We believe that other angular dicarboxylate ligands would be also ideal for building extended coordination polymers, as demonstrated by $4,4^{\prime}$-oxybis(benzoate), with a $118^{\circ}$ angle between the two benzoate groups (Figure 1). The slightly distorted tetrahedral geometry of $\mathrm{Zn}$ (II) atom is completed by four oxygen atoms derived from four different 4,4'-oxybis(benzoate) ligands, with similar $\mathrm{Zn}-\mathrm{O}$ distances shorter than those found in bis(4,4'-bipyridine)zinc(II) succinate [4]. Each 4,4'-oxybis(benzoate) ligand coordinates four zinc(II) atoms through its two carboxylate groups in a bis-bidentate fashion leading to a three-dimensional framework (Figure 2).

\section{EXPERIMENTAL}

The title compound was obtained from a hydrothermal synthesis by reacting zinc nitrate, $4,4^{\prime}-$ oxybis(benzoic) acid and sodium hydroxide in a 1:1:2 molar ratio at $180^{\circ} \mathrm{C}$ for 60 hours. $\mathrm{CH}$ analysis: $\mathrm{C}$ $52.29, \mathrm{H} 2.51 \%$. Calc. for $\mathrm{C}_{14} \mathrm{H}_{8} \mathrm{O}_{5} \mathrm{Zn}$ : C $52.40, \mathrm{H} 2.45 \%$. 
Table 1. Crystal data for zinc(II) 4,4'-oxybis(benzoate)

\begin{tabular}{llll}
\hline Empirical formula & $\mathrm{C}_{14} \mathrm{H}_{8} \mathrm{O}_{5} \mathrm{Zn}$ & Formula weight & 321.57 \\
Crystal system & Monoclinic & Space group & $P 2_{\mathrm{l}} / c$ \\
Unit cell dimensions & $a=7.355(3) \AA$ & $\beta$ & $102.64(2)^{\circ}$ \\
& $b=16.034(5) \AA$ & Volume & $1216.9(11) \AA^{3}$ \\
$Z$ & $c=10.575(8) \AA$ & $T$ & $293 \mathrm{~K}$ \\
$\mu, \mathrm{mm}^{-1}$ & 4 & $D_{\text {exptl }} \mathrm{g} \mathrm{cm}^{-3}$ & 1.755 \\
$\theta$ range & 2.034 & $F(000)$ & 648 \\
Independent reflections & $2.4-27.0^{\circ}$ & Reflections with $\gg 2 \sigma(I)]$ & 1965 \\
Goodness-of-fit on $F^{2}$ & $1.04\left(R_{\text {int }}=0.040\right)$ & Parameters refined & 182 \\
$R$ (all data) & 0.124 & Final $R[D 2 \sigma(I)]$ & 0.050 \\
Weighting scheme, $w$ & {$\left[\sigma^{2}+(0.078 P)^{2}+0.8449 P\right]^{-1}$ where $P=\left(F_{\mathrm{o}}^{2}+2 F_{\mathrm{c}}^{2}\right) / 3$} & $-0.46-0.42 e \AA^{-3}$ \\
Programs & $O R T E P-I I[5], S H E L X-97[6]$ & CCDC deposition no. & 185137 \\
\hline
\end{tabular}

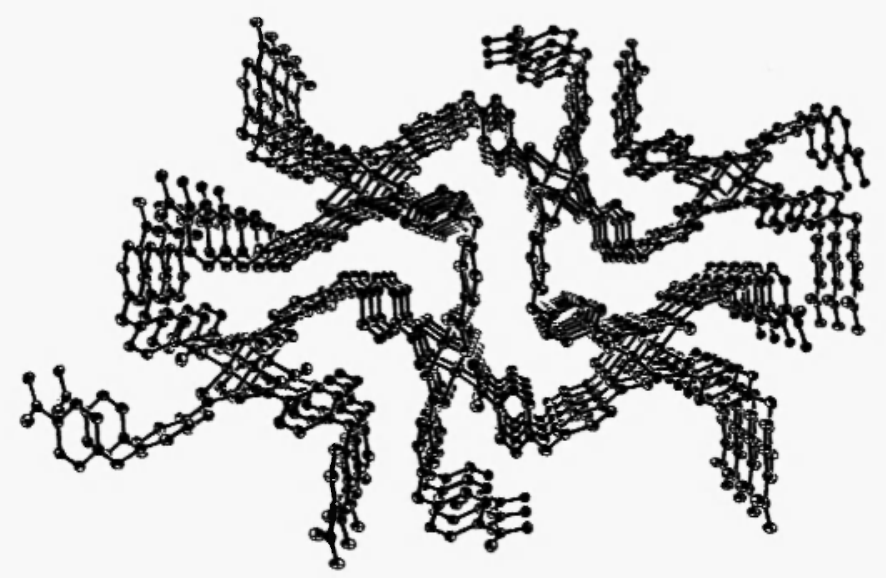

Figure 2. Perspective view of the structure along $a$ axis.

\section{ACKNOWLEDGMENTS}

We thank Xiamen University (Y07024) for supporting this work.

\section{REFERENCES}

1 M. Eddaoudi, D. B. Moler, H. Li, B. Chen, T. M. Reineke, M. O'Keeffe and O. M. Yaghi, Acc. Chem. Res., 34 (2001) 319.

2 M. Eddaoudi, J. Kim, J. B. Wachter, H. K. Chae, M. O'Keeffe and O. M. Yaghi, J. Am. Chem. Soc., 123 (2001) 4368.

3 B. Moulton, J. Lu, A. Mondal and M. J. Zaworotko, Chem. Commun., (2001) 863.

4 J. Tao, Y. L. Fu, S. L. Zheng, X. M. Chen and S. W. Ng, Main Group Met. Chem., 24 (2001) 289.

5 C. K. Johnson, ORTEP-II. Report ORNL-5138. Oak Ridge National Laboratory, Oak Ridge, Tennessee, USA (1976).

6 G. M. Sheldrick, SHELX-97. Program for Crystal Structure Analysis (Release 97-2). University of Göttingen, Germany (1997). 